Multiple other factors (eg age, ethnicity, having English as first language, weekend admission, distance of home from hospital) were not significantly related to pre-admission behaviour.

Conclusions The majority of admitted adult CAP cases presented directly to hospital, but a significant minority had preadmission NHS contact. Efforts to reduce CAP mortality should primarily be directed at in-hospital care.

\begin{tabular}{|c|c|c|c|c|c|}
\hline \multicolumn{2}{|c|}{ Symptoms } & $\begin{array}{l}\text { Total } \\
\mathrm{n}(\%)\end{array}$ & $\begin{array}{l}\text { No pre-admission } \\
\text { NHS contact } \\
n(\%)\end{array}$ & $\begin{array}{l}\text { Pre-admission } \\
\text { NHS contact } \\
n(\%)\end{array}$ & $P$ value \\
\hline \multirow[t]{2}{*}{ Sputum } & Yes & $30(68)$ & $15(50)$ & $15(50)$ & 0.044 \\
\hline & No & $14(38)$ & $12(86)$ & 2 (14) & \\
\hline \multirow{4}{*}{$\begin{array}{l}\text { CURB65 } \\
\text { score }\end{array}$} & 0 & $8(18)$ & $4(50)$ & $4(50)$ & 0.038 \\
\hline & 1 & & & & \\
\hline & $\begin{array}{l}1-2 \\
3\end{array}$ & $\begin{array}{l}20(46) \\
13 \quad(30)\end{array}$ & $\begin{array}{l}16(80) \\
7(54)\end{array}$ & $\begin{array}{l}4(20) \\
6(46)\end{array}$ & \\
\hline & $4 / 5$ & $3(6)$ & $0(0)$ & $3(100)$ & \\
\hline \multirow{4}{*}{$\begin{array}{l}\text { Duration } \\
\text { of illness }\end{array}$} & $1-2$ days & $6(13)$ & $5(83)$ & $1(17)$ & 0.028 \\
\hline & 3-7 days & $25(57)$ & $18(72)$ & 7 (28) & \\
\hline & 8-14 days & $3(7)$ & $0(0)$ & $3(100)$ & \\
\hline & $>14$ days & 10 (23) & $4(40)$ & $6(60)$ & \\
\hline
\end{tabular}

\section{P243 INFLUENZA A OUTBREAK IN A UK RESPIRATORY CENTRE}

A Aujayeb, S West, S Waugh, J Samuel, A Russel, R Fagg, S Gray, C Walton, G Meachery; Freeman Hospital, Newcastle Upon Tyne, England

\subsection{6/thoraxjnl-2013-204457.395}

Introduction In March 2013, 12 patients on a single ward in a tertiary respiratory transplant centre contracted influenza within 72 hours. There was no corresponding community outbreak. Staff with symptoms went off sick. Trust policies outlining respiratory infection and isolation existed but there were no guidelines for this specific novel situation. We found no published reports of such an event in England.

Methods Patients quickly developed pyrexias and respiratory symptoms. All had throat swabs and blood cultures. Influenza A, H3N2 variant, was identified. A team of infection control and respiratory physicians, nurses and managers met regularly to implement these measures:

- Closure of ward and cohorting of bays

- Ward avoidance for non-essential personnel and anyone with symptoms

- Cancellation of non-essential procedures

- Strict hand hygiene and use of PPE and FFP3 masks

- Stockage of oseltamivir for treatment for all affected high risk staff and patients and prophylaxis offered to all ward patients and exposed high risk staff.

- No crossover of ward staff to transplant patients.

- Contact tracing of all immunocompromised patients on ward up to one week and all high risk patients 48 hours prior to the index case; advice on prophylaxis and their GPs contacted.

- Writing an information sheet for staff and GPs

- Increased and terminal ward cleaning

Results On the respiratory ward, 151 bed days were lost and 53 on two other wards. Fourteen patients (including two on another ward) had positive swabs for H3N2. There were 27 symptomatic staff members; 15 had swabs, two were positive. All patients and two staff members were given treatment oseltamivir. Fourteen patients and two staff members had prophylaxis.

No influenza complications or deaths occurred.

The department staff had $45 \%$ influenza vaccination uptake in 2012/2013. All affected patients had been vaccinated.

Conclusions Containment, pathogen identification, prompt treatment and contact tracing were priorities, to limit number of individuals affected. This is widely applicable.

Our departmental staff vaccination rate is below Department of Health targets. Importance of vaccination needs emphasising, whilst recognising that vaccine effectiveness against all laboratory-confirmed influenza in primary care is $51 \%$ for $2012 / 2013$.

\section{P244 ACCURACY OF ADMISSION DIAGNOSIS OF PNEUMONIA}

${ }^{1}$ Lc Price, ${ }^{2} \mathrm{Ms}$ Anwar, ${ }^{3} \mathrm{~K}$ Srikanthan, ${ }^{2} \mathrm{~A}$ Bercusson, ${ }^{1} \mathrm{E}$ Williams, ${ }^{1} \mathrm{E}$ Prior, ${ }^{3} \mathrm{~A}$ Owusu-Ageyi, ${ }^{1} \mathrm{H}$ Umpleby, ${ }^{1} \mathrm{M}$ Berry; ${ }^{1}$ St Mary's Hospital, London, UK; ${ }^{2}$ Hammersmith Hospital, London, UK; ${ }^{3}$ Charing Cross Hospital, London, UK

\subsection{6/thoraxjnl-2013-204457.396}

Background The inclusion of 'new infiltrates' on the admission chest radiograph (CXR) is part of the BTS audit tool (1), but it has been reported that up to $37 \%$ of inpatients coded (using the ICD-10 coding system) as pneumonia did not have CXR consolidation (2). We assessed our local audit population for potential reasons for this pneumonia miscoding.

Methods Patients selected by coding for the 2012/13 BTS Pneumonia Audit at hospitals within Imperial Healthcare Trust (Charing Cross (CX), Hammersmith (HH) and St Mary's (SMH) Hospitals) underwent a notes review.

Results Of all patients clinically coded as community-acquired pneumonia (CAP), 88/176 (50\%) had a diagnosis of CAP compatible with the audit criteria, with infiltrates on the admission CXR-in 15 (39\%), 25 (61\%) and $48(50 \%)$ of cases at CX, HH and $\mathrm{SMH}$ respectively.

Of the patients found not to have CAP by the current BTS audit criteria $(\mathrm{n}=88), 47 / 88(53 \%)$ had an abnormal admission CXR not showing CAP. The main abnormalities in these admission CXRs were pulmonary oedema (in 30\%), COPD/bronchiectasis (27\%), malignancy (13\%), interstitial lung disease (ILD) (7\%) and pleural effusions (7\%). In the 88 'non-audit criteria CAP' patients, in combination with symptoms, inflammatory markers (WCC 10.4 (1.7-33) x109 and CRP 84.8 (3.6-381) mg/ L), and the CXR series, the likely diagnosis was felt to be LRTI (35\%), CAP (17\%), HAP (13\%), COPD (10\%), pulmonary oedema (9\%), malignancy (4.5\%), UTI (3\%), and ILD, asthma and effusions (all 2\%), in the 'non-audited CAP' patients.

Of those remaining 41 patients with a normal admission CXR excluded from the audit, 24 patients (59\%) had a repeat CXR within $72 \mathrm{~h}$, of which 5 (21\%) then showed CAP. An additional 12 patients with normal admission CXRs had CT scans performed within $72 \mathrm{~h}, 6$ of which $(50 \%)$ detected consolidation. Thus, of all the patients with a normal admission CXR, 11 (27\%) had CAP that was missed by solely looking at the admission CXR.

Conclusion We confirm earlier findings that coding diagnoses are insufficiently accurate to judge quality of care, but also show that current audit criteria exclude a significant proportion of cases.

\section{REFERENCES}

1. WS Lim, et al. Thorax 2011;66:548-549

2. Ruickbie SV, et al. Thorax 2012;67(Suppl 2):A69 


\section{P245 A DEDICATED PNEUMONIA FOLLOW UP CLINIC-IS IT WORTHWHILE?}

D Komrower, S Iyer, S Kazmi, V Shanker, P Stockton, S Twite, P Malhotra; Whiston Hospital, Prescot, Liverpool, England

\subsection{6/thoraxjnl-2013-204457.397}

Background It is routine clinical practice, based on British Thoracic Society recommendations, to arrange a clinical review and chest $\mathrm{x}$-ray approximately 6 weeks following an episode of pneumonia in most patients. However there is a paucity of published evidence to support or refute this practice.

Objective To determine the clinical and radiological outcomes of patients admitted with an episode of pneumonia.

Methods This was a retrospective analysis of the post-pneumonia clinic database at a large district general hospital over a one year period from May 2011 to April 2012. Data on demographic characteristics, radiological and clinical follow up, and final diagnosis were collected. Follow up data including a new diagnosis of cancer as well as new benign lung disease was available for most (97\%) patients for 12 months after the index episode of pneumonia. Resolution was defined as complete resolution on chest X-ray at 6-8 weeks or $>90 \%$ resolution on chest X-ray, absence of ongoing physical symptoms and signs, and decision to discharge from clinic after discussion at a multidisciplinary radiology meeting.

Results 179 patients were included in the analysis (32 who did not attend for follow up were excluded). One hundred and thirty nine $(78 \%)$ showed resolution and were discharged. Of the $40(22 \%)$ patients that did not show resolution, 16 (9\%) had a new diagnosis made; including $3(1.7 \%)$ lung cancer. One of the identified lung cancers was stage I disease, while the other two were stage IV. New benign diagnoses made on follow up were recurrent aspiration (3), interstitial lung disease (2), pleural effusion (2), allergic bronchopulmonary aspergillosis (1), cryptogenic organising pneumonia (1), functional antibody deficiency (1), lung nodule (1), pleural plaques (1), and pulmonary embolism (1). Males, ever smokers, older patients, and those with a history of pre-existing lung disease (including COPD) had higher rates of non-resolution but the differences were not statistically significant.

Conclusion Following up patients in a dedicated post pneumonia clinic with a repeat chest radiograph and clinical review had a low but significant pick up rate of new respiratory diagnoses. A larger study will be performed to improve risk stratification and enable more selective follow up.

\begin{tabular}{llll}
$\begin{array}{l}\text { Abstract P245 Table 1. Comparison of clinical characteristics } \\
\text { of patients with resolved versus non-resolved pneumonia }\end{array}$ \\
\hline
\end{tabular}

C Molugu, W Falconer, A Singer, A Disney, P Stockton, V Peers, L McCulloch; St Helens and Knowsley Hospitals NHS Trust, Prtescott, Liverpool, UK

\subsection{6/thoraxinl-2013-204457.398}

Introduction For patients admitted to hospital with pneumonia, CURB65 (confusion, Urea $>7$, Respiratory Rate $>30$ and Blood Pressure $<90 / 60$ ) is used to assess severity of the pneumonia (1). MEWS is a score which informs about the physiological status of a patient thus about the patients overall clinical status. One would anticipate CURB65 to be correlated to MEWS however there is no evidence to support correlation particularly on admission. The body's capability to maintain adequate oxygenation when affected by pneumonia is of paramount importance. Oxygen was originally excluded from CURB65 due to non-statistical significance for predicting mortality. We felt CURB65 may not correlate with MEWS on admission hence introduced a new score incorporating oxygen to CURB65 score - CURBO2-65. COPD patient's with SATS $<88 \%$ or Non-COPD with $<94 \%$ would score 1 and any patient receiving supplemental oxygen to maintain their SATS in desired range would score 1 . The maximum score for oxygenation is 2 and maximum score on CURBO2 65 is 7 instead of 5 as in CURB65.

Method We retrospectively analysed all pneumonia patients (COPD and Non-COPD) admitted to hospital for 2 consecutive months (December 2012 and January2013). Data was collected

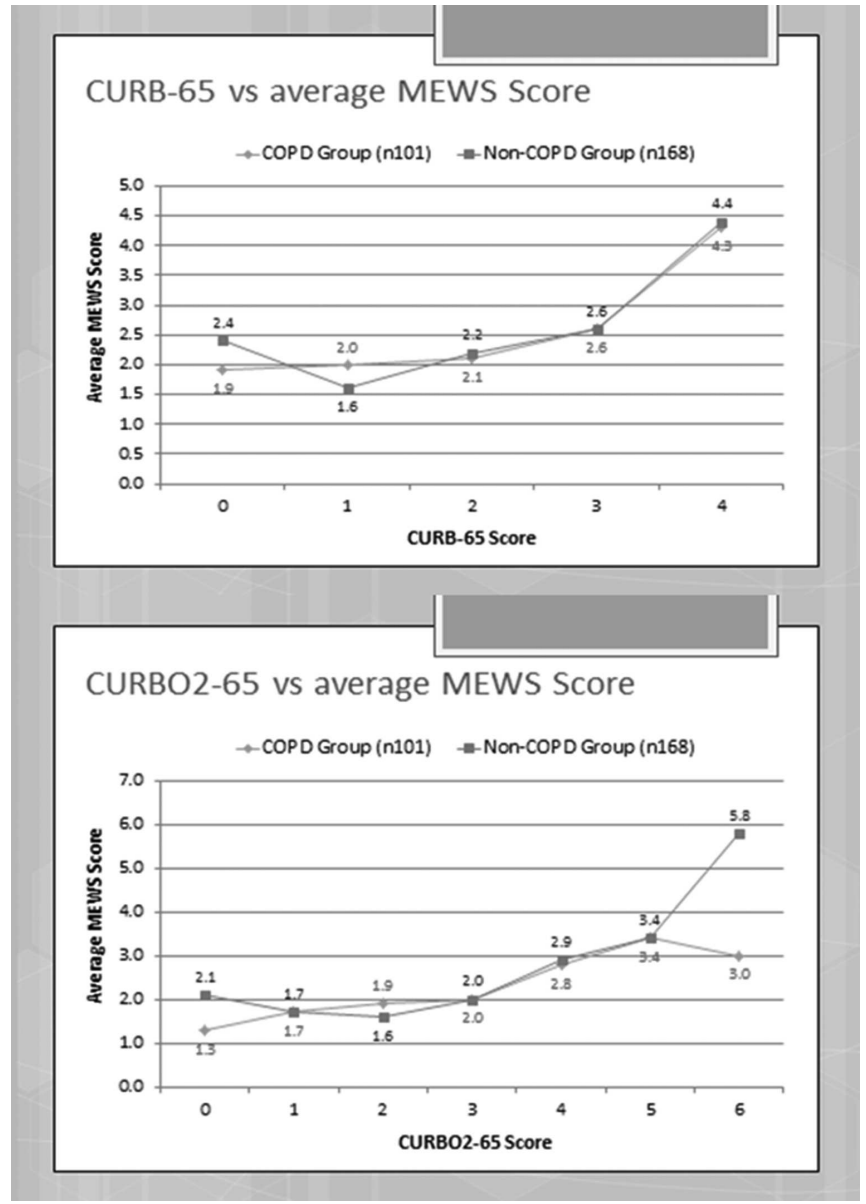

Abstract P246 Figure 1. 